\title{
Why Nanomedicine?
}

\author{
Diego Liberati* \\ National Research Council of Italy, Italy
}

\section{Short Commentary}

To some of us nano medicine looks like the new frontier for therapy, while for others is just a fashionable attitude still poor of true success: who is right? Probably both!

Standard drug therapy is manly assumed in pills or at most in blood at quantities sufficient to let the right amount of active principle reach the target in time, possibly repeated. Often, the target is not diffused in all the body, but localized, like an organ: administration of enough chemical thus means, besides a waste sometimes costly and anyway polluting, an unuseful and often even obnoxious burden to the patient body elsewhere form needed target, overcharging the clearance systems of the patient already stressed by the illness.

Nanoscience and nanotechnology are nowadays mature enough to provide infinitesimal quantities of drug and to be able to administer them, and will improve more and more: the very problem is to convey such a small quantity to the very target instead to having its effect diffused over all the body and thus not sufficient to heal.

Thus a devoted chemical packaging has to be designed and devoted to shield the active principle and not letting it interacting out of target: in this sense, it would just mimicking nature: some hormones for instance travel in blood covered in a cap not allowing them to irritate the capillary vessels (like it unfortunately happens in sudden capillary leaking syndrome, whose unknown cause could thus be exactly some decorticated hormone circulating for some developed reason), then freed at target via one of the beautiful chemo-physical reaction characterizing our physiology.
In the same way, a bright colleague at Padua university, having discovered a nice molecule against cirrhosis, have had the nice idea to embed it in sugar, almost not interacting with blood, but fastly digested by liver, eager of sugar, and where blood come through abundantly, thus exposing just the very target to the drug: it works, as a start up run by her is showing since a few months.

The above is a perfect and fortunate case of course: in general the situation is less easy, but the concept is the same: the cup covering or just embedding the active principle inactivating it temporarily should be resistant to blood and in general transport, when direct administration to target is not possible or at least not easy, and then able to react at target in such a way to release the proper amount of active principle, either or both exploiting simple physiology, like in the beautiful example above, or at least be driven by some exogenous (co-) factors especially designed to make it worth: this is and will be the first challenge in the near future, mainly of chemical nature.

A second challenge could be a less passive transport of the nanodrug through blood to the very target, when standard phyysiology would not be as favorable as for liver: such a further challenge, probably od more physical nature, would finally allow to literally drive, at least in part, the designed nanoparticle embedding the nano drug to the very target in quantities and timing exceeding the blood diffusion ability, thus making even more precise such innovative growing technique of personalizing precision medicine, increasing desired effects while minimizing side effects and polluting waste.
Copyright: (C)2018 Liberati D. This is an open-access article distributed under the terms of the Creative Commons Attribution License, which permits unrestricted use, distribution, and reproduction in any medium, provided the original author and source are credited.
${ }^{*}$ Correspondence to: Diego L, PhD, Research Director National Research Council of Italy, Italy, Tel: +39 3480569317; Email: diego.liberati@polimi.it

Received: September 20, 2018; Accepted: September 26, 2018; Published: September 28, 2018 